\title{
ANNA BERTHA ROENTGEN (1833-1919): LA MUJER DETRAS DEL HOMBRE
}

\author{
Int. Daniela García $\mathbf{P}^{(1)}$, Dr. Cristián García $\mathrm{B}^{(2)}$.
}

1. Interna Facultad de Medicina, Pontificia Universidad Católica de Chile.

2. Departamentos de Radiología y Pediatría. Hospital Clínico Pontificia Universidad Católica de Chile.

\begin{abstract}
Wilhelm Roentgen discovered the $\mathrm{x}$-rays on 1895 and was married to Anna Bertha Roentgen, for forty-seven years. His wife was a big support for him, and very few articles have been written about her. This publication intends to emphasize the importance of Anna in Wilhelm Roentgen's life and work.
\end{abstract}

Key words: Anna Roentgen, Wilhelm Roentgen, Roentgen, Rayos X, Nobel Física.

Resumen: Wilhelm Roentgen fue el descubridor de los rayos $X$ en 1895 y estuvo casado por cuarenta y siete años con Anna Bertha Roentgen. Su esposa fue un pilar fundamental tanto en su vida personal como laboral y muy pocos artículos se han escrito sobre ella. Esta publicación intenta reconstituir lo que fue su vida y su aporte a la obra de Wilhelm Roentgen.

\section{Introducción}

Wilhelm Conrad Roentgen (1845-1923) nació en Lennep, un pequeño pueblo ubicado al oeste de Alemania(1), como único hijo del matrimonio formado por Friederich Conrad Roentgen y Charlotte Constance Frowein. Su padre fue un comerciante textil de clase acomodada y su madre provenía de una familia holandesa de mucho renombre en el área de la manufactura y de la navegación. De esta manera, Wilhelm tuvo una educación muy privilegiada y fue un niño relativamente mimado. Sin embargo, siempre mostró un gran interés por la experimentación ${ }^{(2)}$. Cuando tenía recién tres años de vida, su familia decidió mudarse a Holanda, con los familiares de su madre.

El viernes 8 de noviembre de 1895 Roentgen hizo un descubrimiento trascendental que cambiaría el mundo de la medicina de entonces. Mientras experimentaba en su laboratorio, notó que por medio de un tipo de radiación era capaz de ver a través de

García D. Anna Bertha Roentgen (1833-1919): La mujer detrás del hombre. Rev Chil Radiol 2005; 11: 179-181.

Correspondencia: Int. Daniela García P.

E-mail:mdgarcia@puc.cl materiales, incluyendo la propia piel(3). El denominó este fenómeno «Rayos X», por la naturaleza desconocida de la radiación, y gracias a él ganó el primer Premio Nóbel de Física en el año $1901^{(1,2)}$.

La primera radiografía que tomó Roentgen causó gran impacto en todo el mundo y actualmente es un documento histórico. La imagen corresponde a una mano izquierda y es curioso notar que en su dedo anular llevaba un anillo(4). ¿Quién se prestó para estos extraños experimentos? Nada menos que Anna Bertha Roentgen, su esposa.

A pesar de toda la ayuda que Anna prestó a su marido, muy pocas cosas se han escrito sobre

Figura 1. Anna Bertha Roentgen.

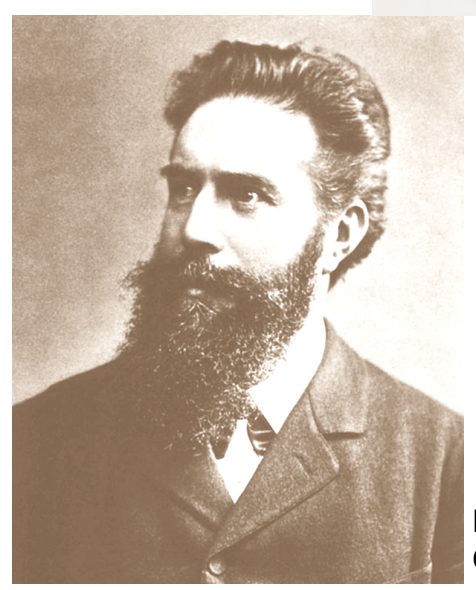

Figura 2. Wihelm Conrad Roentgen. 
ella. Es por ello que nos parece interesante recopilar su historia y sacar del anonimato a esta gran mujer que con su aporte ayudó a construir la medicina que hoy conocemos.

\section{Antecedentes históricos}

Wilhelm y Anna Roentgen contrajeron matrimonio el 7 de julio de 1872 en Apeldoor, Holanda. Se conocieron en Zurich mientras Roentgen trabajaba como ayudante de su Profesor de Física, Augustus Kundt, que fue quien lo inspiró a dedicarse a la ciencia física(5).

Anna era una mujer alta, delgada, muy atractiva y encantadora, hija de Johann Gottfried Ludwig, alemán, gastrónomo y dueño de una pequeña hospedería o café muy popular entre los estudiantes, llamado «Zum Grünen Glas», en español: Hacia el pasto verde. Una tarde de 1866, cuando Roentgen volvía de su trabajo decidió pasar a tomarse un café y allí conoció a Anna(6) ${ }^{(6)}$ Se dice que fue amor a primera vista.

Se comprometieron en 1869, pero el noviazgo no fue fácil. Primero, porque Anna era seis años mayor que Wilhelm, algo muy poco común en esa época y segundo porque el padre de Roentgen se oponía al matrimonio. El tenía ambiciosos planes para su único hijo y se sintió muy decepcionado cuando Wilhelm eligió a alguien de cuna humilde

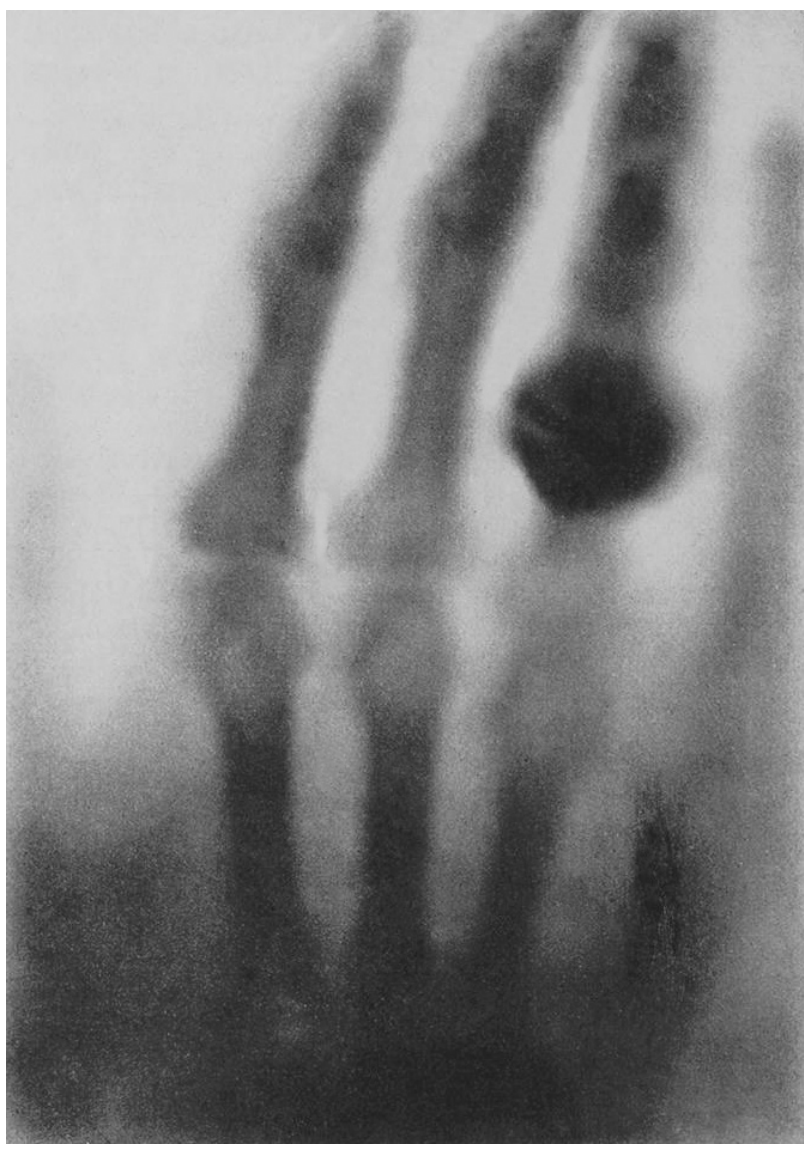

como Anna(6).

Sus primeros años de casados fueron bastante difíciles porque el padre de Roentgen, como represalia, quitó todo apoyo financiero a la nueva familia y como ayudante Wilhelm ganaba muy poco dinero, insuficiente para vivir como estaban acostumbrados a hacerlo ${ }^{(5)}$. Pero eran felices juntos y supieron sobrellevar las adversidades. Con el apoyo de Anna, Roentgen fue haciendo crecer su carrera como físico y académico y en 1894 fue elegido como nuevo rector de la Universidad de Würzburg ${ }^{(7)}$.

Roentgen trabajaba largas horas en sus experimentos, tanto en la Universidad como en el laboratorio que instaló en su casa. Anna tenía gran cariño por su marido y comprendía que Wilhelm necesitaba su espacio para poder concentrarse, pero eso no evitaba que la mayoría del tiempo se sintiera sola. Es por eso que ansiaba mucho poder quedar embarazada y rodearse de hijos que la acompañaran. Pero los años pasaron, y Anna nunca pudo cumplir su sueño.

En 1887 una tragedia sacudió la familia de Anna, su único hermano falleció dejando huérfana a la pequeña Josephine Bertha Ludwig, de sólo seis años. Wilhelm y Anna decidieron adoptarla y así Anna pudo cumplir el anhelo de ser madre ${ }^{(5)}$.

La tarde del viernes 8 de noviembre de 1895, Anna escuchó la llamada de su marido y acudió de inmediato. Wilhelm le solicitó que pusiera su mano en un extraño aparato. Tuvo que estar sin moverse por más de quince minutos y luego Roentgen le mostró la que sería la primera radiografía ${ }^{(8)}$. Se podían ver todos los huesos de su mano y además se distinguía su anillo de compromiso. Está descrito que su reacción, al igual que la de mucha gente de la época, fue una mezcla de fascinación y temor, ya que el hecho de poder distinguir todos sus huesos la hacía sentirse extrañamente cercana a la muerte ${ }^{(9)}$.

La salud de Anna no era buena, sufría constantemente de cólicos renales y otros males aue se fueron aaravando con el transcurso de los

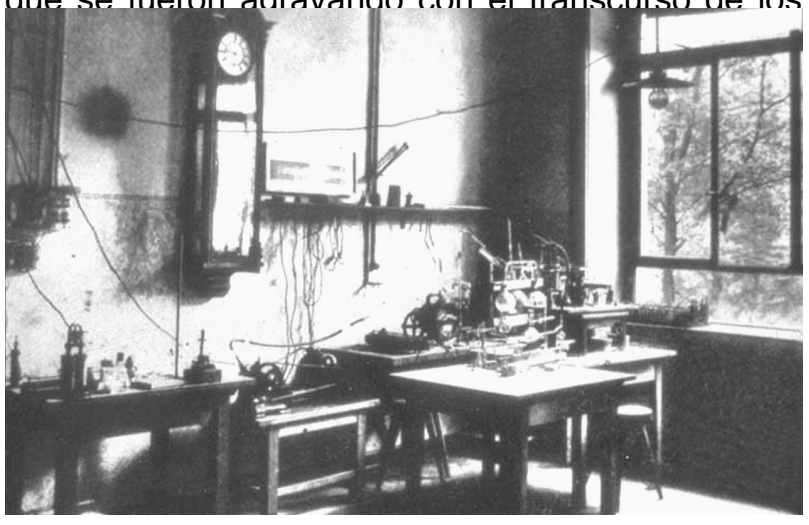

Figura 4. Laboratorio de investigación de Roentgen.

Figura 3. Radiografía de la mano de Anna Roentgen. 
pero Anna finalmente falleció en $1919^{(6)}$. La causa de su muerte no está clara y queda una pregunta sin respuesta: ¿Habrá jugado algún rol su larga exposición a los rayos $X$ en la rústica máquina de Roentgen? Sin embargo, no hay ninguna evidencia científica que apoye esta posibilidad.

Wilhelm nunca se recuperó completamente de la muerte de su mujer y falleció cuatro años después, en Munich, producto de un cáncer del recto $^{(10)}$. Fue enterrado junto a Anna en el Cementerio de Huyesen ${ }^{(5)}$.

Roentgen fue una persona muy reconocida en su época. No sólo por su gran descubrimiento, sino que también por su calidad como persona. Era honesto y siempre actuó con rectitud. Ni siquiera quiso tener los derechos de su descubrimiento porque argumentaba que la única manera de que las investigaciones siguieran creciendo era que los rayos $X$ fueran libres. Sin duda que la presencia de Anna estuvo detrás de muchas de estas decisiones.
1. Eisenberg RL. Radiology. An Illustrated history. Mosby Year Book 1992, St. Louis, MI, USA.

2. Etter LE. Some historical data relating to the discovery of the roentgen rays. AJR 1946; 56: 220-231.

3. Biomedical Imaging Overview. www.whitaker.org

4. Inventor x-rays. Mary Bellis, www. inventors.about. com

5. Wilhelm Conrad Roentgen (1845-1923), the discovery of $x$ rays and perinatal diagnosis. Peter M Dunn. Department of Child Health, University of Bristol, Southmead Hospital, Southmead, Bristol BS10 5NB, UK.

6. Women in Radiology. www.ecr.org/conferences.

7. Traveling with the atom. Glen E. Rodgers.

8. Kraft E, Finby N. Wilhelm Conrad Roentgen (18451923). Discoverer of X-ray. NY State J Med 1974; 74 : 2066-2070.

9. The history of the x-rays. www.umw.edu.

10. Wilhelm Roentgen. www.bibliotecasvirtuales.com

\section{Bibliografía}

